\title{
Observers of Fuzzy Descriptor Systems with Time-Delays
}

\author{
Hongbiao Fan, Min Meng, and Jun-e Feng \\ School of Mathematics, Shandong University, Jinan 250100, China \\ Correspondence should be addressed to Jun-e Feng; fengjune@sdu.edu.cn
}

Received 25 March 2014; Revised 11 June 2014; Accepted 13 June 2014; Published 6 July 2014

Academic Editor: Elena Braverman

Copyright (C) 2014 Hongbiao Fan et al. This is an open access article distributed under the Creative Commons Attribution License, which permits unrestricted use, distribution, and reproduction in any medium, provided the original work is properly cited.

\begin{abstract}
For discrete fuzzy descriptor systems with time-delays, the problem of designing fuzzy observers is investigated in this paper. Based on an equivalent transformation, discrete fuzzy descriptor systems with time-delays are converted into standard discrete systems with time-delays. Then, via linear matrix inequality (LMI) approach, both delay-dependent and delay-independent conditions for the existence of fuzzy state observers are obtained. Finally, two numerical examples are provided to illustrate the proposed method.
\end{abstract}

\section{Introduction}

For many practical engineering systems, increased productivity has led to new operating conditions, which are more challenging. Such conditions would affect system's performance. To improve efficiency, a number of methods have been proposed, such as fault-tolerant control [1], fault detection, and isolation [2]. As far as we know, most practical systems are nonlinear, and it has been proved that any smooth nonlinear system can be accurately presented by TakagiSugeno (T-S) models, which were firstly presented by Takagi and Sugeno in 1985 [3]. Therefore, a lot of attention has been attracted by them and some important results have been obtained $[4,5]$. On the other hand, in many real systems, a nature phenomenon is after-effect. Because of the continuously expanded physical settings and capabilities, the common point-to-point communication form does not work well in modern industry any longer. So it is necessary to seek a new platform. Due to comprehensive diagnosis, low cost, and so on, online communication was introduced, and more and more networks are considered in control loops. However, since the communication media possesses timesharing, time-delays can not be avoided in the control loops. In addition, time-delays often cause instability and affect system's performance. Therefore, many researchers have investigated time-delayed systems [6-8]. However, there are few papers referring to discrete-time fuzzy systems simultaneously including singularity and time-delays.
Interconnected systems, which are described as different names in [9], have been concerned in many researches. Increasing attention is paid to both theory and practical use of interconnected systems. So its fundamental theory and applications have involved a wide field during recent years. In real world, there are also many interconnected systems, such as large electric networks, electric power systems, and different types of societal systems. One such system consists of a series of independent subsystems, but all subsystems are interrelated with some goals. Recently, for the stability and stabilization of interconnected systems, a number of methods have been used $[10,11]$. For a class of uncertain nonlinear descriptor systems with input saturation, [12] considered robust stabilization. Furthermore, with mode-dependent timevarying delays, uncertain discrete-time switched systems were considered to design $l_{2}-l_{\infty}$ filter in [13]. Indeed, T$S$ fuzzy model is also a kind of interconnected systems, and this paper focuses on studying T-S fuzzy models. When the parameters and structure of a system are not known, a fuzzy model can be used. T-S model, which is usually applied to describe a nonlinear complex system, is one of the most common types of fuzzy models. A T-S fuzzy model includes many fuzzy rules and the consequent part of each rule is in fact a local model. A large number of problems about T-S fuzzy model have been considered.

Utilizing the approach of linear matrix inequality (LMI), [14] investigated $D$-stability and nonfragile control for T-S fuzzy discrete-time descriptor systems with multiple delays. 
Subject to stochastic perturbation and time-varying delay, the passivity and passification problems for T-S fuzzy descriptor systems were studied in [15]. There are also some advanced methods to deal with T-S fuzzy time-delayed systems. For example, a linear lower dimensional model was used to approximate the original discrete-time fuzzy system with time-delays in [16], and the model approximation was casted into a sequential minimization problem with LMI constraints. Distributed fuzzy filters were designed for a class of sensor networks, which were described by discrete-time T-S fuzzy systems with time-varying delays and multiple probabilistic packet losses, in [17]. About stability analysis and stabilization for a class of discrete-time T-S fuzzy systems with time-varying state delay, a novel delay-partitioning method was developed in [18] and a stability condition, which is much less conservative than most existing results, was derived by the new idea. Taking advantage of similar delaypartitioning approach, [19] analyzed dissipativity of T-S fuzzy time-delayed descriptor systems. Also for a class of discretetime T-S fuzzy time-delay systems, the problem of reliable filter design with strict dissipativity was considered in [20] and a sufficient condition of reliable dissipativity analysis was proposed. Additionally, filter matrices can be obtained by solving a convex optimization problem. This paper focuses on designing observers for discrete-time fuzzy descriptor systems with time-delays via LMI approach, which is often used. Furthermore, we try to investigate the corresponding problems with delay-partitioning method in our next work.

For a system, the control is often based on state feedback. However, all states of a system are not always available. At this time, it seems very important to estimate system states. So many scholars begin to focus on the problem of designing observers and filters. For example, a proportional multipleintegral observer was investigated for fuzzy chaotic model with unknown input in [21]. As for descriptor time-delayed systems with Markovian jump, [22] discussed designing linear memoryless observers. And a method of designing delay-dependent $H_{\infty}$ filters for singular systems with timedelays was considered in [23]. For fuzzy systems, considerable attention should be paid to fuzzy observers and some achievements have been earned. In [24], a T-S fuzzy system was firstly transformed into a standard form and then a sliding model fuzzy observer could be constructed. With the approach in [24], it is effective to deal with matched and unmatched uncertainties in fuzzy models. In order to estimate the system state and output disturbance at the same time, Gao and his cooperators tried to design a novel fuzzy observer [25], which considered two cases for the output matrices. When each subsystem has the same output matrices, an augmented fuzzy descriptor system could be constructed. When the output matrices of all subsystems are different, a standard T-S fuzzy system was studied. For each case, T-S fuzzy state-space observers were designed, respectively. Moreover, the observer techniques proposed were applied to the fault estimation. In 2007, Marx et al. gave a method of designing decoupling observers for T-S descriptor systems with unknown inputs [26], where the proposed observer could be used to perform fault diagnosis. It is worth noting that only continuous systems are concerned in most existing works. This paper mainly discusses observer design for discrete-time-delayed descriptor systems with T-S fuzzy model.

The paper is organized as follows. Section 2 introduces the problem to be investigated. In Section 3, both delayindependent and delay-dependent sufficient conditions for the existence of fuzzy state observer are given. For each sufficient condition, two numerical examples are shown in Section 4. At last a short conclusion is included in Section 5.

\section{Problem Formulation}

In this section, we will briefly describe the problem to be studied. Through this paper, the following discrete-timedelayed descriptor system is considered.

Plant form is as follows. THEN

Rule j: IF $\theta_{1}(k)$ is $M_{j 1}, \theta_{2}(k)$ is $M_{j 2}, \ldots$, and $\theta_{p}(k)$ is $M_{j p}$,

$$
\begin{gathered}
E x(k+1)=A_{j} x(k)+A_{d j} x(k-d)+B_{j} u(k) \\
y_{j}(k)=C_{j} x(k) \\
x(k)=\phi(k), \quad k=-d,-d+1, \ldots, 0,
\end{gathered}
$$

where $M_{j s}, \theta_{s}(k)(1 \leq s \leq p)$ are fuzzy sets and premise variables, respectively. $x(k) \in \mathbb{R}^{n}$ is the system state, the measurable output is $y(k) \in \mathbb{R}^{q}$, and $u(k) \in \mathbb{R}^{p}$ represents the control input. The initial condition is denoted by $\phi(k)$. It is assumed that $E \in \mathbb{R}^{n \times n}$ is singular; that is, $\operatorname{rank} E=r<n$. The remaining matrices, $A_{j}, A_{d j}, B_{j}$, and $C_{j}$, are known. Besides, $1 \leq j \leq L$, where $L$ is the number of IF-THEN rules. $d, L, p$ are positive integer numbers.

We make the following assumption.

Assumption 1. Consider

$$
\operatorname{rank}\left[\begin{array}{c}
E \\
C_{j}
\end{array}\right]=n, \quad 1 \leq j \leq L,
$$

so there exists a matrix pair $\left[T_{j} N_{j}\right]$ for each $j \in\{1,2, \ldots, L\}$ such that

$$
T_{j} E+N_{j} C_{j}=I_{n}, \quad 1 \leq j \leq L .
$$

Remark 2. Because of the singularity of matrix $E$ in fuzzy system (1), we give Assumption 1. Condition (2) is the same as that in [27], where another condition is also needed. In this paper, only one is enough.

Now we try to transform system (1) into a form as a standard system. Denote by $X^{+}$the pseudoinverse of a matrix $X$. Then the general solution to (3), with condition (2), is

$$
\left[\begin{array}{ll}
T_{j} & N_{j}
\end{array}\right]=\left[\begin{array}{c}
E \\
C_{j}
\end{array}\right]^{+}+\widetilde{Z}\left(I_{n+q}-\left[\begin{array}{c}
E \\
C_{j}
\end{array}\right]\left[\begin{array}{c}
E \\
C_{j}
\end{array}\right]^{+}\right)
$$

where matrix $T_{j}(1 \leq j \leq L)$ is nonsingular, which can be achieved via designing the arbitrary matrix $\widetilde{Z}$ with appropriate dimension. 
According to equality (3), the first equation of fuzzy system (1) is changed into

$$
\begin{aligned}
x(k+1)= & T_{j} A_{j} x(k)+T_{j} A_{d j} x(k-d)+T_{j} B_{j} u(k) \\
& +N_{j} y_{j}(k+1),
\end{aligned}
$$

where $1 \leq j \leq L$.

Let

$$
h_{j}(\theta(k))=\frac{\prod_{s=1}^{p} M_{j s}\left(\theta_{j}(k)\right)}{\sum_{j=1}^{L} \prod_{s=1}^{p} M_{j s}\left(\theta_{j}(k)\right)}, \quad 1 \leq j \leq L,
$$

with $\theta(k)=\left[\theta_{1}(k) \theta_{2}(k) \cdots \theta_{p}(k)\right] . M_{j s}\left(\theta_{j}(k)\right)$ means the grade of membership of $\theta_{j}(k)$ in $M_{j s}$. Obviously, $0 \leq$ $M_{j s}\left(\theta_{j}(k)\right) \leq 1$. Therefore, $h_{j}(\theta(k)) \geq 0(1 \leq j \leq L)$ and $\sum_{j=1}^{L} h_{j}(\theta(k))=1$ for all $k$. For fuzzy system (1), the final state and output are given as follows:

$$
\begin{array}{r}
x(k+1)=\sum_{j=1}^{L} h_{j}(\theta(k))\left[T_{j} A_{j} x(k)+T_{j} A_{d j} x(k-d)\right. \\
\left.+T_{j} B_{j} u(k)+N_{j} y_{j}(k+1)\right], \\
y(k)=\sum_{j=1}^{L} h_{j}(\theta(k)) C_{j} x(k), \\
x(k)=\phi(k), \quad k=-d,-d+1, \ldots, 0 .
\end{array}
$$

Define

$$
\begin{aligned}
& {\left[\begin{array}{llll}
\widetilde{A}(\theta) & \widetilde{A}_{d}(\theta) & \widetilde{B}(\theta) & C(\theta)
\end{array}\right]} \\
& \triangleq \sum_{j=1}^{L} h_{j}(\theta(k))\left[\begin{array}{lllll}
\widetilde{A}_{j} & \widetilde{A}_{d j} & \widetilde{B}_{j} & C_{j}
\end{array}\right],
\end{aligned}
$$

where $\left[\begin{array}{lll}\widetilde{A}_{j} & \widetilde{A}_{d j} & \widetilde{B}_{j}\end{array}\right]=T_{j}\left[\begin{array}{lll}A_{j} & A_{d j} & B_{j}\end{array}\right]$. Then system (7) can be written as

$$
\begin{gathered}
x(k+1)=\widetilde{A}(\theta) x(k)+\widetilde{A}_{d}(\theta) x(k-d)+\widetilde{B}(\theta) u(k) \\
+\sum_{j=1}^{L} h_{j}(\theta(k)) N_{j} y_{j}(k+1), \\
y(k)=C(\theta) x(k), \\
x(k)=\phi(k), \quad k=-d,-d+1, \ldots, 0 .
\end{gathered}
$$

Through the above analysis, singular fuzzy system (1) is transformed into the ordinary linear system (9), for which the observer will be designed. In this paper, the state observer as in the following form is considered.

Observer rule $j$ : IF $\theta_{1}(k)$ is $M_{j 1}, \theta_{2}(k)$ is $M_{j 2}, \ldots$, and $\theta_{p}(k)$ is $M_{j p}$, THEN

$$
\begin{gathered}
\widehat{x}(k+1)=T_{j} A_{j} \widehat{x}(k)+T_{j} A_{d j} \widehat{x}(k-d)+T_{j} B_{j} u(k) \\
+G_{j}(\hat{y}(k)-y(k))+N_{j} y_{j}(k+1), \\
\hat{y}_{j}(k)=C_{j} \hat{x}(k),
\end{gathered}
$$

where $1 \leq j \leq L, \widehat{x}(k) \in \mathbb{R}^{n}$ is the estimate of state $x(k)$, and matrices $G_{j}(j=1,2, \ldots, L)$ are observation error matrices. Denote by $y(k)$ and $\widehat{y}(k)$ the final output of fuzzy system and fuzzy observer, respectively. With the same weight $h_{j}(\theta(k))$ and notations in system (7) the final estimated state and output of fuzzy observer (10) can be described as

$$
\begin{gathered}
\widehat{x}(k+1)=\widetilde{A}(\theta) \hat{x}(k)+\widetilde{A}_{d}(\theta) \widehat{x}(k-d)+\widetilde{B}(\theta) u(k) \\
+G(\theta)(\widehat{y}(k)-y(k))+\bar{y}(k+1), \\
\hat{y}(k)=C(\theta) \widehat{x}(k),
\end{gathered}
$$

where $\bar{y}(k)=\sum_{j=1}^{L} h_{j}(\theta(k)) N_{j} y_{j}(k)$ and $G(\theta) \triangleq$ $\sum_{j=1}^{L} h_{j}(\theta(k)) G_{j}$.

Thus the problem of this paper focuses on finding matrices $G_{j}(j=1,2, \ldots, L)$ such that model (11) is an observer of system (9).

\section{Main Results}

This section discusses how to design fuzzy state observers for discrete descriptor systems with time-delays. And for existence of fuzzy observer, two different sufficient conditions are derived. We give the Schur complement Lemma first.

Lemma 3 (see [28]). The LMI

$$
\left[\begin{array}{ll}
M & S \\
S^{T} & R
\end{array}\right]>0
$$

where $M=M^{T}, R=R^{T}$ are equivalent to

$$
R>0, \quad M-S R^{-1} S^{T}>0 .
$$

Now a sufficient condition about existence of considered observer, which does not rely on time-delay, is presented by the following theorem.

Theorem 4. Model (10) is a state observer of fuzzy system (1), if there exist common matrices $P>0$ and $Q>0$ and matrices $W_{j}(j=1,2, \ldots, L)$ such that

$$
\begin{gathered}
\Lambda_{i i}<0, \quad i=1,2, \ldots, L, \\
\Lambda_{i j}+\Lambda_{j i}<0, \quad 1 \leq i<j \leq L,
\end{gathered}
$$

where

$$
\Lambda_{i j}=\left[\begin{array}{ccc}
Q-P & 0 & \widetilde{A}_{i}^{T} P+C_{j}^{T} W_{i} \\
* & -Q & \widetilde{A}_{d i}^{T} P \\
* & * & -P
\end{array}\right],
$$


and $*$ denotes matrix entries implied by the symmetry of a matrix through this paper. Furthermore, the observer gain matrices can be obtained as

$$
G_{i}=P^{-1} W_{i}^{T}, \quad 1 \leq i \leq L .
$$

Proof. Define

$$
e(k)=x(k)-\widehat{x}(k) .
$$

From system (9) and observer (11), the error dynamic equation of estimation error $e(k)$ can be derived as

$$
e(k+1)=[\widetilde{A}(\theta)+G(\theta) C(\theta)] e(k)+\widetilde{A}_{d}(\theta) e(k-d) .
$$

Take a Lyapunov function as

$$
V(k)=e^{T}(k) P e(k)+\sum_{i=1}^{d} e^{T}(k-i) Q e(k-i),
$$

where $P, Q>0$; then

$$
\begin{aligned}
\Delta V(k)= & V(k+1)-V(k) \\
= & e^{T}(k+1) P e(k+1)-e^{T}(k) P e(k) \\
& +\sum_{i=1}^{d} e^{T}(k+1-i) Q e(k+1-i) \\
& -\sum_{i=1}^{d} e^{T}(k-i) Q e(k-i) \\
= & e^{T}(k+1) P e(k+1)-e^{T}(k) P e(k) \\
& +e^{T}(k) Q e(k)-e^{T}(k-d) Q e(k-d) \\
= & \left\{[\widetilde{A}(\theta)+G(\theta) C(\theta)] e(k)+\widetilde{A}_{d}(\theta) e(k-d)\right\}^{T} \\
& \times P\left\{[\widetilde{A}(\theta)+G(\theta) C(\theta)] e(k)+\widetilde{A}_{d}(\theta) e(k-d)\right\} \\
& -e^{T}(k) P e(k)+e^{T}(k) Q e(k) \\
& -e^{T}(k-d) Q e(k-d) .
\end{aligned}
$$

Let $\eta(k)=\left[\begin{array}{ll}e^{T}(k) & e^{T}(k-d)\end{array}\right]^{T}$; thus

$$
\Delta V(k)=\eta^{T}(k) \Lambda \eta(k),
$$

where

$$
\Lambda=\left[\begin{array}{ll}
\lambda_{1}(\theta) & \lambda_{2}(\theta) \\
\lambda_{2}^{T}(\theta) & \lambda_{3}(\theta)
\end{array}\right]
$$

while $\lambda_{1}(\theta)=[\widetilde{A}(\theta)+G(\theta) C(\theta)]^{T} P[\widetilde{A}(\theta)+G(\theta) C(\theta)]+$ $Q-P, \lambda_{2}(\theta)=[\widetilde{A}(\theta)+G(\theta) C(\theta)]^{T} P \widetilde{A}_{d}(\theta)$, and $\lambda_{3}(\theta)=$ $\widetilde{A}_{d}^{T}(\theta) P \widetilde{A}_{d}(\theta)-Q$. if

The inequality $\Delta V(k)<0$ holds for all $\eta(k) \neq 0$ if and only

$$
\Lambda<0 .
$$

From the Lyapunov stable theory, if inequality (23) holds, the error system (18) would be asymptotically stable.

On the other hand, according to Lemma 3, inequality (23) is equivalent to

$$
\left[\begin{array}{ccc}
Q-P & 0 & {[\widetilde{A}(\theta)+G(\theta) C(\theta)]^{T} P} \\
* & -Q & \widetilde{A}_{d}^{T}(\theta) P \\
* & * & -P
\end{array}\right]<0 .
$$

With the definition of coefficient matrices in system (9), it can be obtained from inequality (24) that

$$
\sum_{i=1}^{L} \sum_{j=1}^{L} h_{i}(\theta(k)) h_{j}(\theta(k))\left[\begin{array}{ccc}
Q-P & 0 & \left(\widetilde{A}_{i}+G_{i} C_{j}\right)^{T} P \\
* & -Q & \widetilde{A}_{d i}^{T} P \\
* & * & -P
\end{array}\right]<0,
$$

which is equivalent to

$$
\sum_{i=1}^{L} \sum_{j=1}^{L} h_{i}(\theta(k)) h_{j}(\theta(k)) \Lambda_{i j}<0 .
$$

And inequality (26) can be rewritten as

$$
\sum_{i=1}^{L} h_{i}(\theta(k)) \Lambda_{i i}+\sum_{i=1}^{L-1} \sum_{j=i+1}^{L} h_{i}(\theta(k)) h_{j}(\theta(k))\left(\Lambda_{i j}+\Lambda_{j i}\right)<0 .
$$

Taking $W_{i}=G_{i}^{T} P$, it follows that conditions (14) are sufficient to guarantee (26) is correct. Inequality (23) holds and the error system (18) is asymptotically stable.

Obviously, conditions in Theorem 4 are not relevant to delay $d$. Next, we will give another result which depends on time-delay.

Theorem 5. For fuzzy system (1), there is a fuzzy state observer in form of (10) if there exist common matrices $P>0, U>0$, and $R>0$ and matrices $W_{j}(j=1,2, \ldots, L)$ such that

$$
\begin{gathered}
\Psi_{i i}<0, \quad i=1,2, \ldots, L, \\
\Psi_{i j}+\Psi_{j i}<0, \quad 1 \leq i<j \leq L,
\end{gathered}
$$

where

$$
\Psi_{i j}=\left[\begin{array}{cccc}
U-P & 0 & \widetilde{A}_{i}^{T} P+C_{j}^{T} W_{i} & \widetilde{A}_{i}^{T} P+C_{j}^{T} W_{i}-P \\
* & -U & \widetilde{A}_{d i}^{T} P & \widetilde{A}_{d i}^{T} P \\
* & * & -P & 0 \\
* & * & * & -\frac{1}{d} R
\end{array}\right] .
$$


Moreover, the observer gain matrices can be calculated as

$$
G_{i}=P^{-1} W_{i}^{T}, \quad 1 \leq i \leq L
$$

Proof. According to proof of Theorem 4, the dynamic equation of error system is (18). Construct a Lyapunov function as

$V(k)$

$$
\begin{aligned}
= & e^{T}(k) P e(k)+\sum_{l=k-d}^{k-1} e^{T}(l) U e(l) \\
& +\sum_{s=-d+1}^{0} \sum_{l=k-1+s}^{k-1}[e(l+1)-e(l)]^{T} Z[e(l+1)-e(l)],
\end{aligned}
$$

where $P, U, Z>0$. Then

$$
\begin{aligned}
\Delta V(k) & \\
= & V(k+1)-V(k) \\
= & e^{T}(k+1) P e(k+1)-e^{T}(k) P e(k) \\
& +\sum_{l=k-d+1}^{k} e^{T}(l) U e(l)-\sum_{l=k-d}^{k-1} e^{T}(l) U e(l) \\
& +\sum_{s=-d+1}^{0}\left\{\sum_{l=k+s}^{k}[e(l+1)-e(l)]^{T} Z[e(l+1)-e(l)]\right. \\
& -\sum_{l=k-1+s}^{k-1}[e(l+1)-e(l)]^{T} \\
& +d[e(k+1)-e(k)]^{T} Z[e(k+1)-e(k)] \\
= & e^{T}(k+1) P e(k+1)-e^{T}(k) P e(k)+e^{T}(k) U e(k) \\
& -e^{T}(k-d) U e(k-d) \\
& -\sum_{s=-d+1}^{0}\left\{[e(k+s)-e(k-1+s)]^{T}\right. \\
& \times Z[e(k+s)-e(k-1+s)]\}
\end{aligned}
$$

$$
\begin{aligned}
& \stackrel{(18)}{=} e^{T}(k) \omega_{1}(\theta) e(k)+e^{T}(k-d) \omega_{2}(\theta) e(k-d) \\
& +e^{T}(k) \omega_{3}(\theta) e(k-d)+e^{T}(k-d) \omega_{3}^{T}(\theta) e(k) \\
& -\sum_{s=-d+1}^{0}[e(k+s)-e(k-1+s)]^{T} \\
& \times Z[e(k+s)-e(k-1+s)],
\end{aligned}
$$

where

$$
\begin{aligned}
\omega_{1}(\theta)= & {[\widetilde{A}(\theta)+G(\theta) C(\theta)]^{T} P[\widetilde{A}(\theta)+G(\theta) C(\theta)] } \\
& -P+U+\omega_{4}(\theta), \\
\omega_{2}(\theta)= & \widetilde{A}_{d}^{T}(\theta) P \widetilde{A}_{d}(\theta)-U+d \widetilde{A}_{d}^{T}(\theta) Z \widetilde{A}_{d}(\theta), \\
\omega_{3}(\theta)= & {[\widetilde{A}(\theta)+G(\theta) C(\theta)]^{T} P \widetilde{A}_{d}(\theta) } \\
& +d[\widetilde{A}(\theta)+G(\theta) C(\theta)-I]^{T} Z \widetilde{A}_{d}(\theta), \\
\omega_{4}(\theta)= & d[\widetilde{A}(\theta)+G(\theta) C(\theta)-I]^{T} \\
& \times Z[\widetilde{A}(\theta)+G(\theta) C(\theta)-I] .
\end{aligned}
$$

Define $\eta(k)=\left[\begin{array}{ll}e^{T}(k) & e^{T}(k-d)\end{array}\right]^{T}$; then

$$
\begin{aligned}
& \Delta V(k)= \eta^{T}(k) \Psi \eta(k) \\
&-\sum_{s=-d+1}^{0}[e(k+s)-e(k-1+s)]^{T} \\
& \quad \times Z[e(k+s)-e(k-1+s)] \\
& \leq \eta^{T}(k) \Psi \eta(k),
\end{aligned}
$$

where

$$
\Psi=\left[\begin{array}{ll}
\omega_{1}(\theta) & \omega_{3}(\theta) \\
\omega_{3}^{T}(\theta) & \omega_{2}(\theta)
\end{array}\right] .
$$

If

$$
\Psi<0
$$

then $\Delta V(k)<0$ and the error system (18) is asymptotically stable.

By Lemma 3, inequality (36) is equivalent to 


$$
\left[\begin{array}{cccc}
U-P & 0 & {[\widetilde{A}(\theta)+G(\theta) C(\theta)]^{T} P} & {[\widetilde{A}(\theta)+G(\theta) C(\theta)-I]^{T}} \\
* & -U & \widetilde{A}_{d}^{T}(\theta) P & \widetilde{A}_{d}^{T}(\theta) \\
* & * & -P & 0 \\
* & * & * & -\frac{1}{d} Z^{-1}
\end{array}\right]<0
$$

Define $J \triangleq \operatorname{diag}[I, I, I, P]$. Pre- and postmultiplying inequality (37) by matrix $J$, we can obtain

$$
\left[\begin{array}{cccc}
U-P & 0 & {[\widetilde{A}(\theta)+G(\theta) C(\theta)]^{T} P} & {[\widetilde{A}(\theta)+G(\theta) C(\theta)-I]^{T} P} \\
* & -U & \widetilde{A}_{d}^{T}(\theta) P & \widetilde{A}_{d}^{T}(\theta) P \\
* & * & -P & 0 \\
* & * & * & -\frac{1}{d} P Z^{-1} P
\end{array}\right]<0
$$

Taking $R=P Z^{-1} P$ and $W_{i}=G_{i}^{T} P$, it is derived that inequality (38) is equivalent to

$$
\sum_{i=1}^{L} \sum_{j=1}^{L} h_{i}(\theta(k)) h_{j}(\theta(k)) \Psi_{i j}<0
$$

That is

$$
\sum_{i=1}^{L} h_{i}(\theta(k)) \Psi_{i i}+\sum_{i=1}^{L-1} \sum_{j=i+1}^{L} h_{i}(\theta(k)) h_{j}(\theta(k))\left(\Psi_{i j}+\Psi_{j i}\right)<0 .
$$

Combining with inequalities (28), we can know that inequality (39) holds and condition (36) is correct. Thus the error system (18) is asymptotically stable.

Remark 6. It is easy to see that Theorem 5 implies Theorem 4. In fact, with $\varphi_{i j} \triangleq\left[P \widetilde{A}_{i}+W_{i}^{T} C_{j}-P P \widetilde{A}_{d i} 0\right]^{T}(i, j=$ $1,2, \ldots, L)$, we have

$$
\Psi_{i j}=\left[\begin{array}{cc}
\Lambda_{i j} & \varphi_{i j} \\
* & -\frac{1}{d} R
\end{array}\right]
$$

where matrices $\Psi_{i j}$ and $\Lambda_{i j}$ are defined in Theorems 5 and 4 , respectively. So, according to Lemma 3, inequalities (28) are equivalent to $\Lambda_{i i}+d \varphi_{i i} R^{-1} \varphi_{i i}^{T}<0(i=1,2, \ldots, L)$ and $\Lambda_{i j}+\Lambda_{j i}+(d / 2)\left(\varphi_{i j}+\varphi_{j i}\right) R^{-1}\left(\varphi_{i j}+\varphi_{j i}\right)^{T}<0(1 \leq i<j \leq L)$, respectively. Since $R>0$, from inequalities (28) we can obtain that $\Lambda_{i i}<0(i=1,2, \ldots, L)$ and $\Lambda_{i j}+\Lambda_{j i}<0(1 \leq i<$ $j \leq L)$. Therefore, the conditions of Theorem 5 are stronger than those of Theorem 4 . On the other hand, if there do not exist compatible solutions to inequalities (28) for some fuzzy descriptor system, we may design its observer based on Theorem 4.

Remark 7. For fuzzy state-space systems, the results of this paper can also be applied. Choosing $E=I_{n}$, a discrete fuzzy state-space system can be derived from fuzzy system (1) without time-delays. Let $T_{j}=I_{n}, N_{j}=0$, and $\widetilde{A}_{d j}=0(1 \leq j \leq$ $L)$. Then, the presented observer in [4] can also be obtained from model (10).

At the end, we give an algorithm to design observer for fuzzy descriptor system (1).

Algorithm 8. The following steps are introduced to determine observer (10) for fuzzy descriptor system (1) according to Theorem 4.

(1) Verify condition (2). If it holds, then go to step 2. Otherwise, stop.

(2) According to formulation (4), solve (3) and guarantee that matrix $T_{j}(1 \leq j \leq L)$ is nonsingular. Then transform fuzzy system (1) into system (9) with solutions to (3).

(3) Solve LMIs (14). If they are solvable, go to step 4. Otherwise, stop.

(4) Give fuzzy observer (10), with $G_{i}=P^{-1} W_{i}^{T}(1 \leq i \leq$ $L)$.

The steps of designing observer for fuzzy descriptor system (1) based on Theorem 5 are similar to Algorithm 8 and omitted here.

Remark 9. In this paper, we firstly consider observer design for fuzzy discrete systems with singularity and time-delays simultaneously. Compared with existing work [27], the singularity of fuzzy system can be easily eliminated under only one simple assumption. Finally, both delay-dependent and delayindependent conditions are derived for obtaining observer gain matrices. 


\section{Examples}

In this section, two numerical examples are provided. The first one is to design a fuzzy observer according to Theorem 4 and the second one illustrates Theorem 5.

Example 10. Consider a discrete fuzzy descriptor system with time-delays as follows.

Plant Rule 1: IF $x_{1}(k)$ is $M_{1}\left(x_{1}(k)\right)$, THEN

$$
\begin{gathered}
E x(k+1)=A_{1} x(k)+A_{d 1} x(k-d)+B_{1} u(k), \\
y_{1}(k)=C_{1} x(k), \\
x(k)=\phi(k), \quad k=-d,-d+1, \ldots, 0 .
\end{gathered}
$$

Plant Rule 2: IF $x_{1}(k)$ is $M_{2}\left(x_{1}(k)\right)$, THEN

$$
\begin{gathered}
E x(k+1)=A_{2} x(k)+A_{d 2} x(k-d)+B_{2} u(k), \\
y_{2}(k)=C_{2} x(k), \\
x(k)=\phi(k), \quad k=-d,-d+1, \ldots, 0,
\end{gathered}
$$

where

$$
\begin{gathered}
E=\left[\begin{array}{ll}
1 & 0 \\
0 & 0
\end{array}\right], \quad A_{1}=\left[\begin{array}{cc}
0 & 1 \\
-1 & 1
\end{array}\right], \quad A_{2}=\left[\begin{array}{cc}
0 & -1 \\
-1 & 1
\end{array}\right], \\
A_{d 1}=\left[\begin{array}{cc}
-0.1 & 0 \\
0.2 & 0.1
\end{array}\right], \quad A_{d 2}=\left[\begin{array}{cc}
0 & 0.1 \\
-0.2 & 0
\end{array}\right], \\
B_{1}=\left[\begin{array}{l}
0 \\
1
\end{array}\right], \quad B_{2}=\left[\begin{array}{c}
0 \\
-1
\end{array}\right], \quad C_{1}=\left[\begin{array}{l}
0 \\
1
\end{array}\right]^{T}, \\
C_{2}=\left[\begin{array}{l}
1 \\
1
\end{array}\right]^{T},
\end{gathered}
$$

and membership functions are

$$
\begin{aligned}
& M_{1}\left(x_{1}(k)\right) \triangleq 1-\frac{x_{1}^{2}(k)}{2.25}, \\
& M_{2}\left(x_{1}(k)\right) \triangleq \frac{x_{1}^{2}(k)}{2.25} .
\end{aligned}
$$

Orbits of membership functions for Rules 1 and 2 are shown in Figure 1.

Coefficient matrices of fuzzy descriptor system are given, and it can be easily verified that matrices $E$ and $C_{j}(j=1,2)$ satisfy condition (2). Based on formulation (4), a solution to (3) is shown as

$$
\begin{array}{ll}
T_{1}=\left[\begin{array}{cc}
1 & 0 \\
0 & -1
\end{array}\right], & N_{1}=\left[\begin{array}{l}
0 \\
1
\end{array}\right], \\
T_{2}=\left[\begin{array}{cc}
1 & 0 \\
-1 & -1
\end{array}\right], & N_{2}=\left[\begin{array}{l}
0 \\
1
\end{array}\right] .
\end{array}
$$

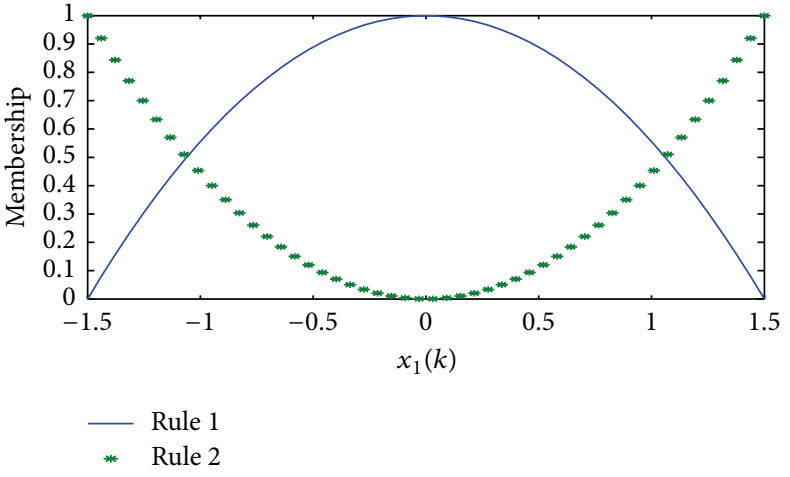

FIgURE 1: Membership functions.

Then fuzzy descriptor system is converted into the following ordinary system:

$$
\begin{gathered}
x(k+1)=\widetilde{A}(k) x(k)+\widetilde{A}_{d}(k) x(k-d)+\widetilde{B} u(k) \\
+\sum_{j=1}^{2} M_{j}\left(x_{1}(k)\right) N_{j} y_{j}(k+1), \\
y(k)=\widetilde{C}(k) x(k), \\
x(k)=\phi(k), \quad k=-d,-d+1, \ldots, 0,
\end{gathered}
$$

where

$$
\begin{aligned}
& {\left[\begin{array}{llll}
\widetilde{A}(k) & \widetilde{A}_{d}(k) & \widetilde{B}(k) & \widetilde{C}(k)
\end{array}\right]} \\
& \quad=\sum_{j=1}^{2} M_{j}\left(x_{1}(k)\right)\left[\begin{array}{llll}
T_{j} A_{j} & T_{j} A_{d j} & T_{j} B_{j} & C_{j}
\end{array}\right] .
\end{aligned}
$$

By using MATLAB LMI Toolbox and solving LMIs in Theorem 4, we obtain

$$
\begin{array}{cc}
P=\left[\begin{array}{cc}
227.2273 & -25.4600 \\
-25.4600 & 61.9384
\end{array}\right], & W_{1}=\left[\begin{array}{c}
-199.6323 \\
57.1439
\end{array}\right]^{T}, \\
Q=\left[\begin{array}{cc}
39.4769 & 3.5449 \\
3.5449 & 27.3497
\end{array}\right], & W_{2}=\left[\begin{array}{c}
181.1576 \\
-32.4363
\end{array}\right]^{T},
\end{array}
$$

so

$$
G_{1}=\left[\begin{array}{c}
-0.8126 \\
0.5886
\end{array}\right], \quad G_{2}=\left[\begin{array}{c}
0.7742 \\
-0.2054
\end{array}\right] .
$$

The state trajectories of discrete-time-delay fuzzy descriptor system are shown in Figure 2 and their estimations are depicted by Figure 3. On the other hand, from Figure 4 we know that the error system is asymptotically stable.

Example 11. Consider the fuzzy system in Example 10. Assume that time-delay $d=2$, and take the same solution to 


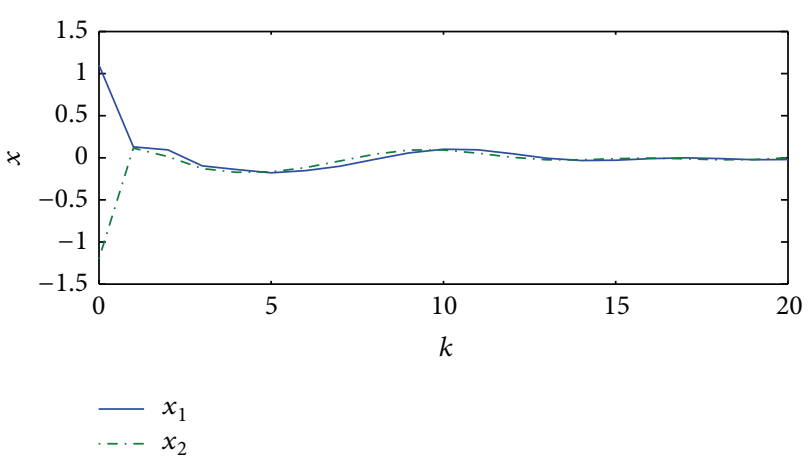

Figure 2: State of the fuzzy descriptor system.

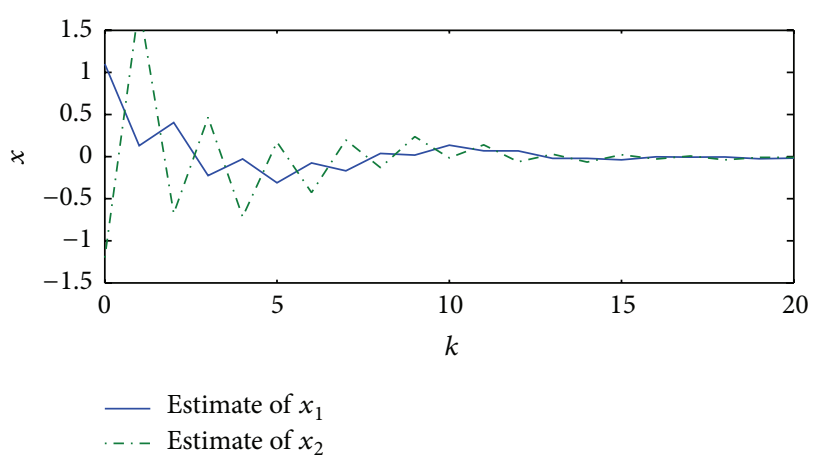

Figure 3: State of the fuzzy observer.

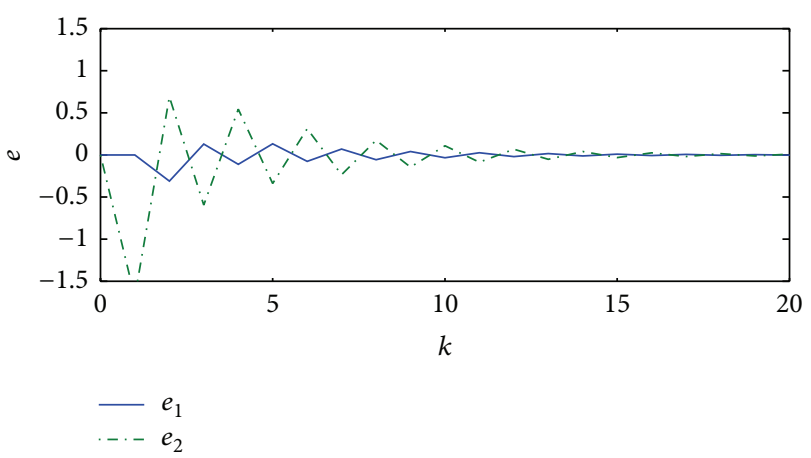

FIGURE 4: Error system.

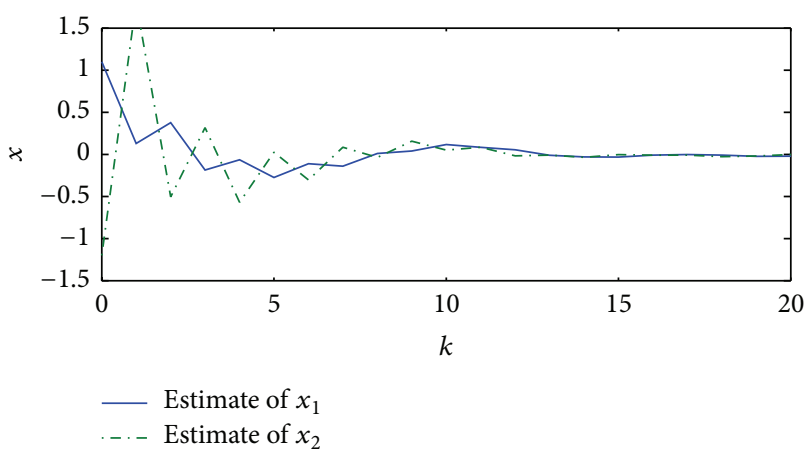

Figure 5: State of the fuzzy observer.

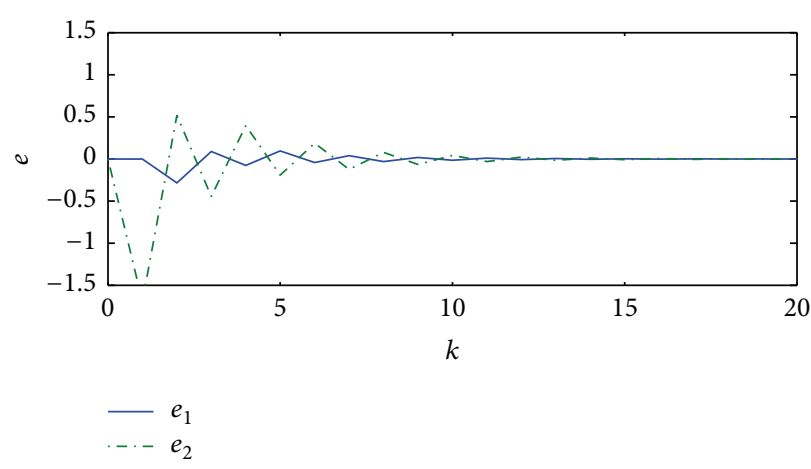

FIGURE 6: Error system.

(3) as that in Example 10. According to Theorem 5 and using MATLAB LMI Toolbox, we obtain

$$
\begin{gathered}
P=\left[\begin{array}{cc}
0.8184 & -0.0655 \\
-0.0655 & 0.1411
\end{array}\right], \quad W_{1}=\left[\begin{array}{c}
-0.7240 \\
0.1516
\end{array}\right]^{T}, \\
U=\left[\begin{array}{cc}
0.0658 & 0.0075 \\
0.0075 & 0.0657
\end{array}\right], \quad W_{2}=\left[\begin{array}{c}
0.7072 \\
-0.0666
\end{array}\right]^{T}, \\
R=\left[\begin{array}{cc}
14.4443 & -2.7589 \\
-2.7589 & 5.1602
\end{array}\right] .
\end{gathered}
$$

Based on matrices $P$ and $W_{j}(j=1,2)$, we can derive

$$
G_{1}=\left[\begin{array}{c}
-0.8295 \\
0.6896
\end{array}\right], \quad G_{2}=\left[\begin{array}{c}
0.8582 \\
-0.0735
\end{array}\right] \text {. }
$$

State trajectories of fuzzy system are the same as those in Figure 2, and Figure 5 is state response of fuzzy observer. Furthermore, from the error system depicted in Figure 6, we know that error converges to zero asymptotically.

Remark 12. From these two examples above, it is obvious that error in Figure 6 converges faster than that in Figure 4. In summary, fuzzy observer designed according to Theorem 5 is better; that is, the convergence time of error is shorter. However, if there is no appropriate observer for some discrete fuzzy descriptor system based on Theorem 5, we may design its observer depending on Theorem 4.

\section{Conclusion}

This paper has discussed how to design fuzzy observers for discrete descriptor systems with time-delays. According to a simple transformation, the singularity of considered fuzzy systems has been eliminated. Then two sufficient conditions for the existence of fuzzy observer have been derived. Notably, one condition depends on time-delays and the other does not. Finally, different fuzzy observers have been designed for the same discrete-time-delayed descriptor system. By comparison, the fuzzy observer depending on delay condition is better. Additionally, our future work will deal with fuzzy time-delayed descriptor systems by using delaypartitioning method. 


\section{Conflict of Interests}

The authors declare that there is no conflict of interests regarding the publication of this paper.

\section{Acknowledgments}

This work was partially supported by NNSF of China (61174141, 61374025), Research Awards Young and MiddleAged Scientists of Shandong Province (BS2011SF009, BS2011DX019), and Excellent Youth Foundation of Shandong Province (JQ201219).

\section{References}

[1] M. Blanke and J. Schroder, Diagnosis and Fault-Tolerant Control, Springer, Berlin, Germany, 2003.

[2] M. Kinnaert, "Fault diagnosis based on analytical models for linear and nonlinear systems-a tutorial," in Preprints of the 5th IFAC Symposium on Fault Detection, Supervision and Safety for Technical Processes SAFEPROCESS, pp. 37-50, 2003.

[3] T. Takagi and M. Sugeno, "Fuzzy identification of systems and its applications to modeling and control," IEEE Transactions on Systems, Man and Cybernetics, vol. 15, no. 1, pp. 116-132, 1985.

[4] X. Ma, Z. Sun, and Y. He, "Analysis and design of fuzzy controller and fuzzy observer," IEEE Transactions on Fuzzy Systems, vol. 6, no. 1, pp. 41-51, 1998.

[5] K. Tanaka and M. Sano, "On the concepts of regulator and observer of fuzzy control systems," in Proceedings of the 3 rd IEEE Conference on Fuzzy Systems, pp. 767-772, 1994.

[6] Y. He, M. Wu, J. She, and G. Liu, "Parameter-dependent Lyapunov functional for stability of time-delay systems with polytopic-type uncertainties," IEEE Transactions on Automatic Control, vol. 49, no. 5, pp. 828-832, 2004.

[7] Y. Li, S. Tong, and L. Yongming, "Observer-based adaptive fuzzy Back stepping control for strict-feedback stochastic nonlinear systems with time delays," International Journal of Innovative Computing, Information and Control, vol. 8, no. 12, pp. 81038114, 2012.

[8] S. Xu, J. Lam, S. Huang, and C. Yang, " $\mathrm{H}_{8}$ model reduction for linear time-delay systems: continuous-time case," International Journal of Control, vol. 74, no. 11, pp. 1062-1074, 2001.

[9] C. H. Lee, T. H. S. Li, and F. C. Kung, "On the robust stability for continuous large-scale uncertain systems with time delays in interconnections," Journal of the Chinese Institute of Engineers, vol. 17, no. 4, pp. 577-584, 1994.

[10] X. Yan and G. Dai, "Decentralized output feedback robust control for nonlinear large-scale systems," Automatica, vol. 34, no. 11, pp. 1469-1472, 1998.

[11] H. Trinh and M. Aldeen, "A comment on decen tralized stabilization of large scale interconnected systems with delays," IEEE Transactions on Automatic Control, vol. 40, no. 5, pp. 914916, 1995.

[12] Y. Ma, Z. Na, and X. Zhong, "Decentralized robust control for uncertain nonlinear descriptor large-scale composite systems with input saturation," International Journal of Innovative Computing, Information and Control, vol. 9, no. 10, pp. 3991-4000, 2013.

[13] G. Zong, L. Hou, and J. Li, "A descriptor system approach to $l_{2}-l_{\infty}$ filtering for uncertain discrete-time switched system with mode-dependent time-varying delays," International Journal of Innovative Computing, Information and Control A, vol. 7, no. 5, pp. 2213-2224, 2011.

[14] F. Li, P. Shi, L. Wu, and X. Zhang, "Fuzzy-model-based dstability and non fragile control for discrete-time descriptor systems with multiple delays," IEEE Transactions on Systems, no. 99, p. 1, 2013.

[15] C. Han, L. Wu, P. Shi, and Q. Zeng, "Passivity and pacification of T-S fuzzy descriptor systems with stochastic perturbation and time delay," IET Control Theory \& Applications, vol. 7, no. 13, pp. 1711-1724, 2013.

[16] L. Wu, X. Su, P. Shi, and J. Qiu, "Model approximation for discrete-time state-delay systems in the T-S fuzzy framework," IEEE Transactions on Fuzzy Systems, vol. 19, no. 2, pp. 366-378, 2011.

[17] X. Su, L. Wu, and P. Shi, "Sensor networks with random link failures: distributed filtering for T-S fuzzy systems," IEEE Transactions on Industrial Informatics, vol. 9, no. 3, pp. 1739-1750, 2013.

[18] L. Wu, X. Su, P. Shi, and J. Qiu, "A new approach to stability analysis and stabilization of discrete-time T-S fuzzy timevarying delay systems," IEEE Transactions on Systems, Man, and Cybernetics B: Cybernetics, vol. 41, no. 1, pp. 273-286, 2011.

[19] C. Han, L. Wu, P. Shi, and Q. Zeng, "On dissipativity of TakagiSugeno fuzzy descriptor systems with time-delay," Journal of the Franklin Institute, vol. 349, no. 10, pp. 3170-3184, 2012.

[20] X. Su, P. Shi, L. Wu, and M. Basin, "Reliable filtering with strict dissipativity for T-S fuzzy time-delay systems," IEEE Transactions on Cybernetics, no. 99, 2014.

[21] T. Youssef, M. Chadli, H. R. Karimi, and M. Zelmat, "Chaos synchronization based on unknown input proportional multipleintegral fuzzy observer," Abstract and Applied Analysis, vol. 2013, Article ID 670878, 11 pages, 2013.

[22] Y. Li, Y. Kao, and J. Xie, "Robust guaranteed cost observer design for singular Markovian jump time-delay systems with generally incomplete transition probability," Abstract and Applied Analysis, vol. 2014, Article ID 832891, 11 pages, 2014.

[23] Z. Li and S. Zhu, "Delay-dependent $H_{\infty}$ filtering for singular time-delay systems," Discrete Dynamics in Nature and Society, vol. 2011, Article ID 760878, 20 pages, 2011.

[24] R. Palm and P. Bergsten, "Sliding mode observer for a Takagi Sugeno fuzzy system," in proceedings of the 9th IEEE International Conference on Fuzzy Systems, pp. 665-670, May 2000.

[25] Z. Gao, X. Shi, and S. X. Ding, "Fuzzy state/disturbance observer design for T-S fuzzy systems with application to sensor fault estimation," IEEE Transactions on Systems, Man, and Cybernetics B Cybernetics, vol. 38, no. 3, pp. 875-880, 2008.

[26] B. Marx, D. Koenig, and J. Ragot, "Design of observers for Takagi-Sugeno descriptor systems with unknown inputs and application to fault diagnosis," IET Control Theory and Applications, vol. 1, no. 5, pp. 1487-1495, 2007.

[27] Z. Wang, Y. Shen, X. Zhang, and Q. Wang, "Observer design for discrete-time descriptor systems: an LMI approach," Systems and Control Letters, vol. 61, no. 6, pp. 683-687, 2012.

[28] S. Boyd, L. Ghaoui, E. Feron, and V. Balakrishnan, Linear Matrix Inequalities in System and Control Theory, Society for Industrial and Applied Mathematics, Philadelphia, Pa, USA, 1994. 


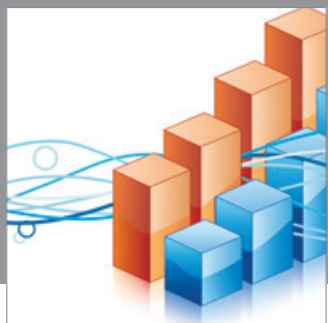

Advances in

Operations Research

mansans

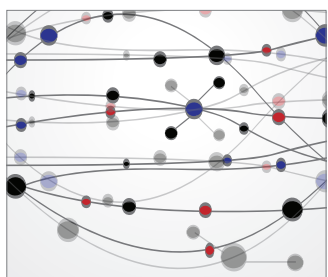

The Scientific World Journal
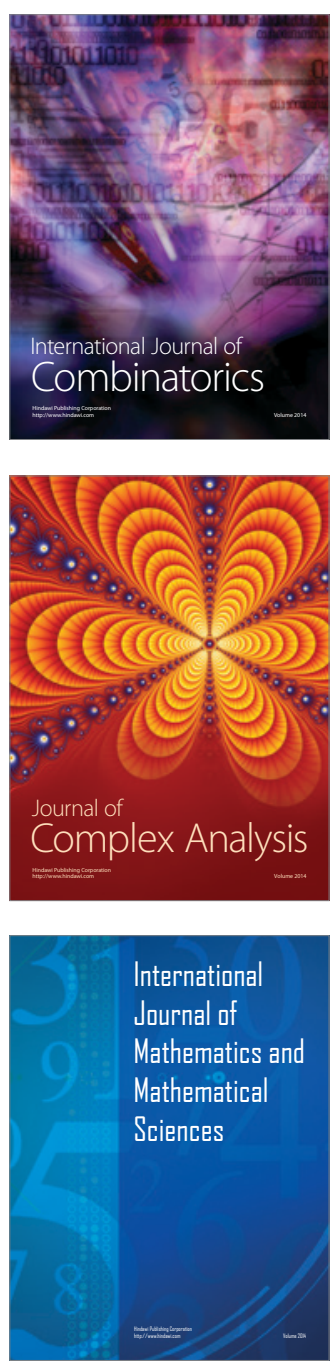
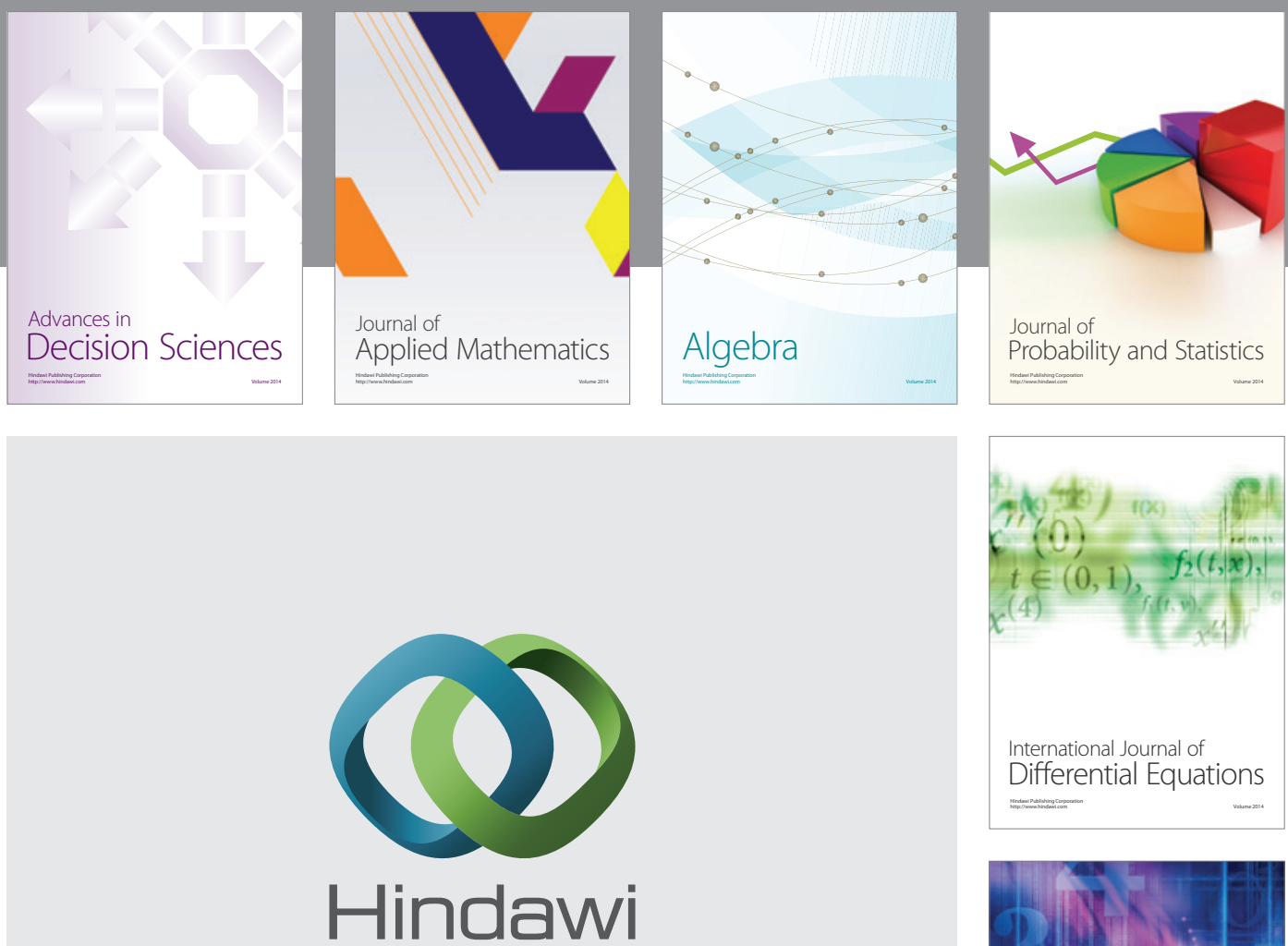

Submit your manuscripts at http://www.hindawi.com
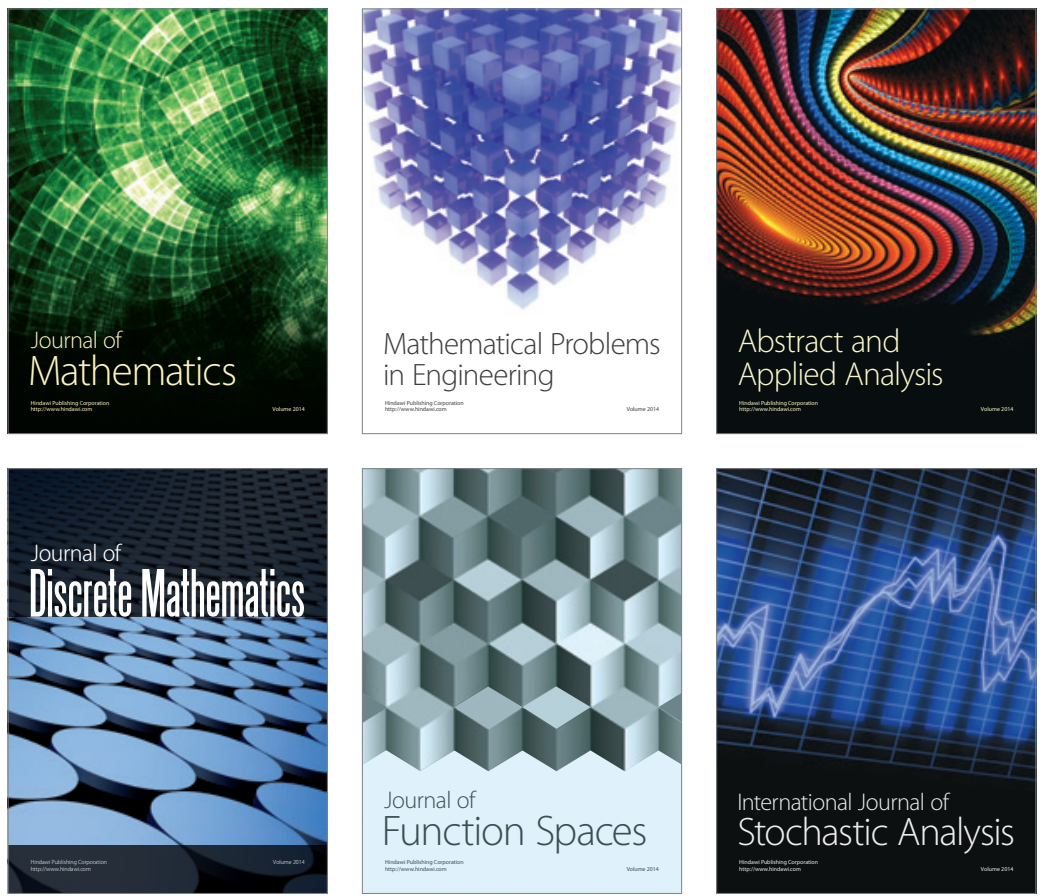

Journal of

Function Spaces

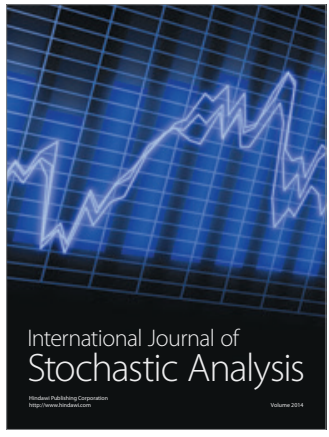

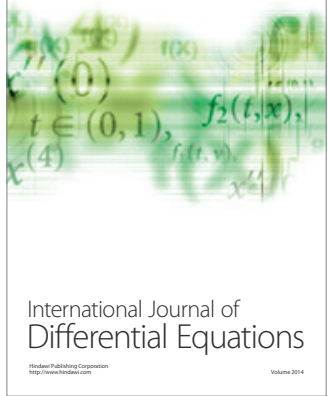
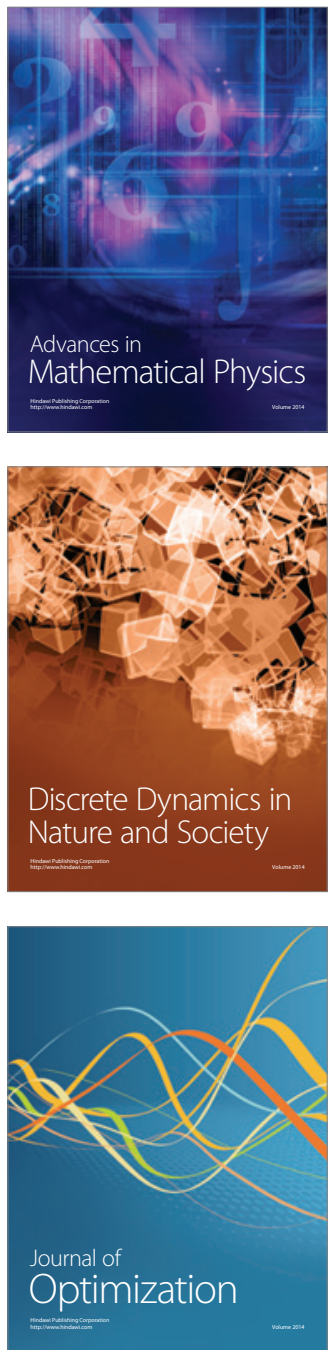